\title{
Atomic static displacements and their effect on the short range order in alloys
}

\author{
Z.Gurskii, Yu.Khokhlov \\ Institute for Condensed Matter Physics, National Academy of Sciences \\ of Ukraine, 1 Svientsitskii St., UA-290011 Lviv, Ukraine
}

Received January 26, 1998

The influence of local atomic static displacements (ASD) on the short range order formation in binary alloys is investigated within the microscopic theory. Explicit expression for the binary correlation function Fourier components $\left\langle\rho_{\boldsymbol{k}} \rho_{-\boldsymbol{k}}\right\rangle$ is obtained by the collective variables method. The theoretical results are illustrated by numerical calculations performed for disordered alloys of the K-Cs and Ca-Ba systems. A drastic effect of the ASD on the $\left\langle\rho_{\boldsymbol{k}} \rho_{-\boldsymbol{k}}\right\rangle$-function behaviour in the first Brillouin zone is observed. The ASD smooth the dispersion of the $\left\langle\rho_{\boldsymbol{k}} \rho_{-\boldsymbol{k}}\right\rangle$-function. Negative values of the short-range order parameter on the first coordination sphere indicate a trend to the ordering in alloys of the systems investigated. The ASD are shown to favour the ordering tendency. The theoretical conclusions concerning the temperature influence on the short range order parameter perfectly agree with the experimental data from the treatment of the X-ray diffuse scattering in binary alloys.

Key words: atomic static displacements, short-range order, disordered alloy, binary correlation function, free energy

PACS: 05.70.Ce; 65.50.+m

\section{Introduction}

It is well known that the short-range order (SRO) in binary alloys is caused by the difference in effective interactions between ions of two kinds, namely, see $[1,2]$

$$
\left\langle\rho_{\boldsymbol{k}} \rho_{-\boldsymbol{k}}\right\rangle \sim V_{2}(\boldsymbol{k})
$$

where $\left\langle\rho_{\boldsymbol{k}} \rho_{-\boldsymbol{k}}\right\rangle$ and $V_{2}(\boldsymbol{k})$ are the Fourier components of the binary correlation function and the ordering potential

$$
V_{2}(R)=V_{A A}(R)+V_{B B}(R)-2 V_{A B}(R),
$$

respectively, index $j=A, B$ denotes a sort of an alloy component. The SRO influences different alloy characteristics, such as electrical conductivity, magnetic 
and galvanomagnetic properties [1,3]. A close correlation between the SRO and mechanical properties is also observed [3]. That is why, investigation of the factors which alter the SRO in alloys is an urgent problem. Atomic static displacements (ASD) could be regarded as one of such factors.

Formation of metal solid solutions is accompanied by the arising of local lattice distortions. The latter are characterised by the ASD with respect to the ideal mean lattice sites. The ASD have a drastic effect on the X-ray (neutron) diffuse scattering [1]. They determine a lattice parameter dependence on alloy concentration. However, the mutual influence of the ASD and the SRO on each other has not been investigated yet within the microscopic theory.

Study of the ASD effect on the SRO formation is the purpose of the present paper. It is organized as follows. Derivation of explicit expressions for the alloy free energy by the collective variables method is given in section 2. Special attention is paid to the original moments of the considered approach. Behaviour of the binary correlation function Fourier components in the Brillouin zone principal directions is analysed in section 3 . The theory is illustrated by numerical calculations carried out for the alloys of $\mathrm{K}-\mathrm{Cs}$ and $\mathrm{Ca}-\mathrm{Ba}$ systems. Calculations have been performed for two cases:

1) with the ASD taken into account;

2) in the rigid lattice approximation, that is without the ASD.

The influence of the ASD on the SRO parameter $\alpha_{R}$ values is also considered in section 3. The dependence of $\alpha_{R}$ on temperature and alloy concentration is presented. Conclusions in section 4 complete the paper.

\section{The binary alloy free energy}

Consider a substitutional binary alloy. Atoms of two kinds $A$ and $B$ are placed arbitrarily on $N$ crystal lattice sites. Their configuration is given by the set $\left\{\sigma_{\boldsymbol{R}}\right\}$ of numbers $\sigma_{\boldsymbol{R}}$ which equal +1 if the site $\boldsymbol{R}$ is occupied by the $A$-kind atom and equal -1 otherwise. The alloy Hamiltonian within the pair interatomic interaction approximation, after summing over electron states $[2,4]$ has the form:

$$
\begin{aligned}
H\left(\sigma_{\boldsymbol{R}}\right) & =\frac{1}{2 N} \sum_{\mathbf{R}_{i}, \mathbf{R}_{j}}\left\{\sum _ { \mathbf { q } } \left[\left(V_{A A}(\mathbf{q}) \frac{1+\sigma_{\mathbf{R}_{i}}}{2} \frac{1+\sigma_{\mathbf{R}_{j}}}{2}+V_{A B}(\mathbf{q}) \frac{1+\sigma_{\mathbf{R}_{i}}}{2} \frac{1-\sigma_{\mathbf{R}_{j}}}{2}+\right.\right.\right. \\
& \left.\left.\left.+V_{B A}(\mathbf{q}) \frac{1-\sigma_{\mathbf{R}_{i}}}{2} \frac{1+\sigma_{\mathbf{R}_{j}}}{2}+V_{B B}(\mathbf{q}) \frac{1-\sigma_{\mathbf{R}_{i}}}{2} \frac{1-\sigma_{\mathbf{R}_{j}}}{2}\right) e^{i \mathbf{q}\left(\mathbf{R}_{i}-\mathbf{R}_{j}\right)}\right]\right\} .
\end{aligned}
$$

Here $V_{i j}(\boldsymbol{q}), i, j=A, B$ is the Fourier transform of the effective interaction between ions of $i$ and $j$ kinds, $V_{A B}(\boldsymbol{q})=V_{B A}(\boldsymbol{q})$. The explicit expressions for $V_{i j}(\boldsymbol{q})$ are given in $[2,4,5]$. Let us take into account the fact that the local ASD are present in an alloy. Then, the coordinates of the lattice sites are the following ones:

$$
\boldsymbol{R}=\boldsymbol{R}^{0}+\delta \boldsymbol{R}
$$

where $\delta \boldsymbol{R}$ are the ASD with respect to the sites $\boldsymbol{R}^{0}$ of the ideal mean lattice. Assume that $\delta \boldsymbol{R}$ does not depend on the kind of an atom and perform the Fourier 
transformation of $\delta \boldsymbol{R}$ :

$$
\delta \boldsymbol{R}=\frac{1}{\sqrt{N}} \sum_{\mathbf{k} \in B Z}\left[\delta \mathbf{R}_{\mathbf{k}} \exp \left(i \mathbf{k} \boldsymbol{R}^{0}\right)+\delta \boldsymbol{R}_{-\boldsymbol{k}} \exp \left(-i \mathbf{k} \boldsymbol{R}_{j}^{0}\right)\right]
$$

Symbol $\boldsymbol{k} \in B Z$ means that the wave vector $\boldsymbol{k}$ takes $N$ values in the first Brillouin zone (BZ). The ASD $\left(\delta \boldsymbol{R}_{j}\right.$; as well as $\left.\delta \boldsymbol{R}_{\boldsymbol{k}}\right)$ are random quantities in a disordered alloy. Let us separate $\boldsymbol{A}_{\boldsymbol{k}}$, a configurationally independent part of $\delta \boldsymbol{R}_{\boldsymbol{k}}$, by means of the following relationship [5]

$$
\delta \mathbf{R}_{\mathbf{k}}=i \frac{\mathbf{A}_{\mathbf{k}}}{2}\left(\hat{\rho}_{\boldsymbol{k}}-\sqrt{N} \delta_{0, \boldsymbol{k}}\right) \quad \boldsymbol{A}_{-\boldsymbol{k}}=-\boldsymbol{A}_{\boldsymbol{k}} .
$$

Here

$$
\hat{\rho}_{\mathbf{k}}=\frac{1}{\sqrt{N}} \sum_{\mathbf{R}} \sigma_{\mathbf{R}} \exp \left(-i \mathbf{k} \mathbf{R}_{i}{ }^{0}\right) .
$$

is the $\boldsymbol{k}$-th Fourier component of the occupation numbers, $\delta_{0, \boldsymbol{k}}$, the Kronecker symbol. Equations (6) and (7) indicate that the Fourier components of the local lattice distortions are caused by fluctuations of the impurity concentration waves with respect to the average value $C_{B}=N_{B} / N$ where $N_{i}, i=A, B$ is the number of $i$-kind atoms. Component $B$ is regarded as an "impurity". One should emphasize that the approximation (6) works very well in the whole region of $C_{B}$ values $(0<$ $\left.C_{B}<1\right)$ in such alloys where dependence of the mean lattice parameter on $C_{B}$ is close to the linear one $[1,5]$.

Let us expand the factor $\exp (i \boldsymbol{q} R)$ in $(3)$ in power series of the static displacements $\delta \boldsymbol{R}$, restricting ourselves to the square of $\delta \boldsymbol{R}$. The alloy Hamiltonian $H(\sigma)$ (3) with allowance for (4) to (7) in the harmonic approximation [5] takes the form

$$
H\left(\sigma_{\boldsymbol{R}}\right)=H_{0}(\hat{\rho})+\sum_{\mathbf{k} \in B Z}\left[H_{1}\left(\mathbf{k}, \delta \mathbf{A}_{\mathbf{k}}, \hat{\rho}_{\mathbf{k}}\right)+H_{2}\left(\mathbf{k}, \boldsymbol{A}_{\boldsymbol{k}}, \hat{\rho}_{\mathbf{k}}\right)\right],
$$

where

$$
H_{0}(\hat{\rho})=N V_{0}+\sqrt{N} V_{1} \hat{\rho}_{0}+\frac{1}{2} \sum_{\mathbf{k} \in B Z} V_{2}(\mathbf{k}) \hat{\rho}_{\mathbf{k}} \hat{\rho}_{-\mathbf{k}}
$$

is the Hamiltonian of an ideal mean lattice without displacements. The explicit expressions for potentials $V_{0}, V_{1}$ and $V_{2}(\boldsymbol{k})$ are presented in [2,5]. They have the following physical meaning: $V_{0}$ is the part of alloy energy which does not depend on atomic configuration, $V_{1}$ indicates the difference between alloy component atomic characteristics [5] and $V_{2}(\boldsymbol{k})$ is the Fourier transform of the ordering potential.

The addends $H_{1}\left(\boldsymbol{k}, \boldsymbol{A}_{\boldsymbol{k}}, \hat{\rho}_{\boldsymbol{k}}\right)$ and $H_{2}\left(\boldsymbol{k}, \boldsymbol{A}_{\boldsymbol{k}}, \hat{\rho}_{\boldsymbol{k}}\right)$ are linear and quadratic in $\boldsymbol{A}_{\boldsymbol{k}}$ amplitudes, respectively. The explicit equations for them see in [5].

We proceed from the grand partition sum to find the free energy

$$
\tilde{Z}=\operatorname{Tr}_{\left\{\sigma_{R}\right\}} \exp \left\{-\beta\left[H(\sigma)-\sum_{i=A, B} \mu_{i} N_{i}\right]\right\} \text {. }
$$

The following notations are introduced in (10): $\beta=\left(k_{B} T\right)^{-1}$ is the inverse temperature, $\mu_{i}$ - the chemical potentials of the alloy components. Symbol $\operatorname{Tr}_{\left\{\sigma_{R}\right\}}$ in 
(10) means summing over all the possible values of the occupation numbers $\left\{\sigma_{\boldsymbol{R}}\right\}$. One can rewrite equation (10) with a view of (8) and using the rigid ideal lattice of an alloy as a reference system, as follows:

$$
Z=\exp \left[-N \beta \tilde{V}_{0}(\mu)\right] \operatorname{Tr}_{\left\{\sigma_{\boldsymbol{R}}\right\}} \exp \left[-\beta \tilde{V}_{1}(\mu) \sum_{\boldsymbol{R}} \sigma_{\boldsymbol{R}}-\frac{1}{2} \sum_{\boldsymbol{k} \in B Z} \beta \tilde{V}_{2}\left(\boldsymbol{k}, \boldsymbol{A}_{\boldsymbol{k}}\right) \hat{\rho}_{\boldsymbol{k}} \hat{\rho}_{-\boldsymbol{k}}\right] .
$$

Details are given in [5]. Here

$$
\begin{aligned}
& \tilde{V}_{0}(\mu)=V_{0}-\frac{1}{2}\left(\mu_{A}+\mu_{B}\right)+\frac{1}{4} \boldsymbol{A}_{0} \Phi^{(0)} \boldsymbol{A}_{0} \\
& \tilde{V}_{1}(\mu)=V_{1}-\frac{1}{2}\left(\mu_{A}-\mu_{B}\right)+\frac{1}{2} \boldsymbol{P}_{0} \boldsymbol{A}_{0}-\frac{1}{2} \boldsymbol{A}_{0} \Phi^{(0)} \boldsymbol{A}_{0} \\
& \tilde{V}_{2}\left(\boldsymbol{k}, \boldsymbol{A}_{\boldsymbol{k}}\right)=V_{2}(\boldsymbol{k})-\boldsymbol{P}_{\boldsymbol{k}} \boldsymbol{A}_{\boldsymbol{k}}+\frac{1}{2} \boldsymbol{A}_{\boldsymbol{k}} \Phi^{(0)} \boldsymbol{A}_{\boldsymbol{k}}
\end{aligned}
$$

are the addends of the alloy Hamiltonian (8) renormalized by the ASD and

$$
\begin{aligned}
& \boldsymbol{P}_{\boldsymbol{k}}=\frac{1}{4} \sum_{\boldsymbol{G}}\left\{(\boldsymbol{G}-\boldsymbol{k})\left[V_{A A}(\boldsymbol{G}-\boldsymbol{k})-V_{B B}(\boldsymbol{G}-\boldsymbol{k})\right]-\right. \\
& \left.-(\boldsymbol{G}+\boldsymbol{k})\left[V_{A A}(\boldsymbol{G}+\boldsymbol{k})-V_{B B}(\boldsymbol{G}+\boldsymbol{k})\right]\right\}
\end{aligned}
$$

The next notations are accepted in (12)-(15): $\boldsymbol{G}$ are the reciprocal lattice vectors, and $\Phi^{(0)}$ - the force constant matrix of the reference system. The correlated average crystal (CAC) in the rigid lattice approximation is used as a reference system. One can get familiarized with the CAC term value in $[2,6]$. The expression for $\Phi^{(0)}$ is given in [6], also see appendix 2 in [5].

The grand partition sum (11) is calculated by the collective variables (CV) method $[2,7]$. Equation (11) is rewritten in the following way within the CV method $[2,7,8]$.

$$
\tilde{Z}=\exp \left[-N \beta \tilde{V}_{0}(\mu)\right] \int \ldots \int \exp \left[-\frac{1}{2} \beta \sum_{\boldsymbol{k} \in B Z} \tilde{V}_{2}\left(\boldsymbol{k}, \boldsymbol{A}_{\boldsymbol{k}}\right) \rho_{\boldsymbol{k}} \rho_{-\boldsymbol{k}}\right] J(\rho) \prod_{\boldsymbol{k} \in B Z} d \rho_{\boldsymbol{k}}
$$

where

$$
J(\rho)=\operatorname{Tr}_{\left\{\sigma_{\boldsymbol{R}}\right\}} J\left(\rho_{\boldsymbol{k}}, \sigma_{\boldsymbol{R}}\right) \exp \left[-\beta \tilde{V}_{1}(\mu) \sum_{\boldsymbol{R}} \sigma_{\boldsymbol{R}}\right]
$$

is the transition Jacobian to the CV space and

$$
J\left(\rho, \sigma_{\boldsymbol{R}}\right)=\prod_{\boldsymbol{k} \in B Z} \delta\left(\rho_{\boldsymbol{k}}-\frac{1}{\sqrt{N}} \sum_{\boldsymbol{R}} \sigma_{\boldsymbol{R}} \exp (-i \boldsymbol{k} R)\right)
$$

with $\delta$, the Dirac delta function.

The general ideas of the CV method are presented in $[2,7]$. We omit them here and pay attention to the original moments of the given paper. Including potential $\tilde{V}_{1}(\mu)(13)$ into the transition Jacobian (17) is an important feature of 
the approach considered here. It allows one to achieve an adequate description of the alloy physical properties within the simplest Gaussian approximation of the $\mathrm{CV}$ method and the rigid lattice approximation [8].

Calculation of the grand partition sum (16) can be performed analytically in the Gaussian approximation. Details of the consideration are omitted because they are similar to those, given in $[5,8]$. Then the grand potential per one atom equals

$$
\begin{aligned}
& \tilde{F}(T, \mu)=-k_{B} T N^{-1} \ln \tilde{Z}=\tilde{V}_{0}(\mu)-\beta^{-1}\left(\ln 2+M_{0}\right)+\frac{1}{2} \frac{\tilde{V}_{2}(0) M_{1}^{2}}{1+\beta \tilde{V}_{2}(0) M_{2}}+ \\
& +(2 N \beta)^{-1} \sum_{\boldsymbol{k} \in B Z} \ln \left[1+\beta \tilde{V}_{2}\left(\boldsymbol{k}, \boldsymbol{A}_{\boldsymbol{k}}\right) M_{2}\right] .
\end{aligned}
$$

Here

$$
M_{n}=\left.\frac{\partial^{n}}{\partial x^{n}} \ln \cosh x\right|_{x=\beta \tilde{V}_{1}(\mu)} \quad n=0,1,2 \ldots
$$

are cumulants $[2,7]$. It is seen from $(20)$ and $(13)$ that $M_{n}(n=0,1,2)$ are complex functions of temperature, potential $\tilde{V}_{1}$ and alloy component chemical potentials. Equation

$$
C_{A}-C_{B}=\frac{\partial \tilde{F}}{\partial \mu_{B}}-\frac{\partial \tilde{F}}{\partial \mu_{A}}
$$

determines the difference of alloy components chemical potentials at the given alloy concentration. The explicit form for equation (21) is presented in [8].

One has to perform the Legandre transformation

$$
F(T, C)=\tilde{F}(T, \mu)+\sum_{i=A, B} \mu_{i} C_{i}
$$

and solve equation (21) to find the alloy free energy $F(T, C)$ as a function of temperature and component concentration. Then,

$$
\begin{aligned}
& F(T, C)=\tilde{V}_{0}+\frac{1}{2} \frac{\tilde{V}_{2}(0) M_{1}^{2}}{1+\beta \tilde{V}_{2}(0) M_{2}}+\beta^{-1}\left\{\ln 2+M_{0}-\frac{1}{2 N} \sum_{\boldsymbol{k} \in B Z} \ln [1+\right. \\
& \left.\left.+\beta \tilde{V}_{2}\left(\boldsymbol{k}, \boldsymbol{A}_{\boldsymbol{k}}\right) M_{2}\right]\right\}+\left(\beta^{-1} x-\tilde{V}_{1}\right)\left\{M _ { 1 } \left[\frac{1}{1+\beta \tilde{V}_{2}(0) M_{2}}+\right.\right. \\
& \left.\left.+\frac{1}{N} \sum_{\boldsymbol{k} \in B Z} \frac{\beta \tilde{V}_{2}\left(\boldsymbol{k}, \boldsymbol{A}_{\boldsymbol{k}}\right)}{1+\beta \tilde{V}_{2}\left(\boldsymbol{k}, \boldsymbol{A}_{\boldsymbol{k}}\right) M_{2}}\right]+M_{1} M_{2}\left[\frac{\beta \tilde{V}_{2}(0) M_{1}}{1+\beta \tilde{V}_{2}(0) M_{2}}\right]^{2}\right\},
\end{aligned}
$$

where

$$
\begin{aligned}
& \tilde{V}_{0}=\tilde{V}_{0}(\mu)+\frac{1}{2}\left(\mu_{A}+\mu_{B}\right), \\
& \tilde{V}_{1}=\tilde{V}_{1}(\mu)+\frac{1}{2}\left(\mu_{A}-\mu_{B}\right),
\end{aligned}
$$


and $x=\beta \tilde{V}_{1}(\mu)$, see (13), is the solution of the system of equations

$$
\frac{\partial F(T, C)}{\partial \boldsymbol{A}_{\boldsymbol{k}}}=0
$$

and (21). Solution of equation (25) is given in [5]. We present the final result omitting details

$$
A_{\mathbf{k} \lambda}=\sum_{\lambda} \frac{\left(\mathbf{P}_{\mathbf{k}} \epsilon_{\mathbf{k} \lambda}\right)}{\overline{m \omega}_{\mathbf{k} \lambda}^{2}} \varepsilon_{\boldsymbol{k} \lambda}
$$

Here $\varepsilon_{k \lambda}$ and $\bar{\omega}_{\mathbf{k} \lambda}^{2}$ are eigenvectors and eigenvalues of the force constant matrix $\Phi^{(0)}$, respectively, $\lambda=1,2,3$ - the polarization index and

$$
\bar{m}=\sum_{i=A, B} m_{i} C_{i}
$$

is the average ion mass, see $[2,6]$ for details. Analyse result (26). One can conclude from (15) and (26) that the ASD amplitudes $\boldsymbol{A}_{\boldsymbol{k}}$ are small if the pair interatomic potentials $V_{A A}$ and $V_{B B}$ Fourier components are similar: $V_{A A}(q) \approx V_{B B}(q)$. Really, $\boldsymbol{P}_{\boldsymbol{k}} \equiv 0$ at $V_{A A}(q)=V_{B B}(q)$ and then $\boldsymbol{A}_{\boldsymbol{k}}=0$. This conclusion allows one to clear up the nature of the well-known phenomenological Hume-Rothery rules [9] on the microscopic level. Using equations (13), (24) and condition (25) one can prove that

$$
\tilde{V}_{1}=V_{1} \text {. }
$$

It means that the potential $\tilde{V}_{1}$ as well as the cumulants $M n$ (20) do not depend explicitly on the ASD amplitudes $\boldsymbol{A}_{\boldsymbol{k}}$. This result simplifies very much the calculation of the alloy free energy (23). Let us analyse more carefully equation (23) for the alloy free energy. The third term in (23) proportional to $\beta^{-1}$ is entropy $(S)$, while the rest of the terms define the alloy internal energy $(E)$. One can get the next formulae for $E$ and $S$ considering equations (23) and (21) in the high temperature limit: $\beta V_{2}(\boldsymbol{k}) \ll 1$.

$$
\begin{gathered}
E_{i d}=V_{0}+V_{1}\left(C_{A}-C_{B}\right)+\frac{1}{2} V_{2}(0)\left(C_{A}-C_{B}\right)^{2}, \\
S_{i d}=-k_{B} \sum_{i=A, B} C_{i} \ln C_{i} .
\end{gathered}
$$

Equation (29) determines the energy of an average crystal: all the lattice sites are occupied by mean ions which interact via the mean potential.

$$
\bar{v}=v_{A} C_{A}+v_{B} C_{B}
$$

with $v_{i}$ - the potential of an $i$-kind ion. Equation (30) defines the configurational entropy of an ideal binary solution. Thus, the high temperature limit of the CV method Gaussian approximation is equivalent to the well-known W.Bragg - E.Williams theory which ignores the pair atomic correlations. By the way, the difference

$$
\Delta F=F(T, C)-E_{i d}+T S_{i d}
$$

with $F(T, C)(23)$ indicates contribution of the SRO effects to the alloy free energy. 


\section{Pair correlation functions and short-range order in alloys of K-Cs and Ca-Ba systems}

The Fourier components of the binary correlation function are important alloy characteristics. They are needed for the calculation of the X-ray (neutron) diffuse scattering intensity $[1,3]$. Besides, they are related to the SRO parameter

$$
\alpha_{R}=\frac{1}{4 C_{A} C_{B}} \sum_{\boldsymbol{k} \in B Z}\left\langle\rho_{\boldsymbol{k}} \rho_{-\boldsymbol{k}}\right\rangle \exp (i \boldsymbol{k} \boldsymbol{R}) .
$$

Here $\alpha_{R}$ is the value of the SRO parameter on the R-coordination sphere, $\left\langle\rho_{\boldsymbol{k}} \rho_{-\boldsymbol{k}}\right\rangle-$ the Fourier components of the binary correlation function. Calculation of $\left\langle\rho_{\boldsymbol{k}} \rho_{-\boldsymbol{k}}\right\rangle$ does not face any difficulties within the Gaussian approximation of the CV method $[2,7]$

$$
\left\langle\rho_{\boldsymbol{k}} \rho_{-\boldsymbol{k}}\right\rangle=-\frac{\partial \ln \tilde{Z}}{\partial\left(\frac{1}{2} \beta \tilde{V}_{2}(\boldsymbol{k})\right)}=\left[1+\beta \tilde{V}_{2}\left(\boldsymbol{k}, \boldsymbol{A}_{\boldsymbol{k}}\right) M_{2}\right]^{-1} .
$$

Potential $\tilde{V}_{2}\left(\boldsymbol{k}, \boldsymbol{A}_{\boldsymbol{k}}\right)(14)$, renormalized by the ASD, takes the form [10]

$$
\tilde{V}_{2}\left(\boldsymbol{k}, \boldsymbol{A}_{\boldsymbol{k}}\right)=V_{2}(\boldsymbol{k})-\frac{1}{2} \sum_{\lambda} \frac{\left(\boldsymbol{P}_{\boldsymbol{k}} \varepsilon_{\boldsymbol{k} \lambda}\right)^{2}}{\bar{m}_{\mathbf{k} \lambda}^{2}}
$$

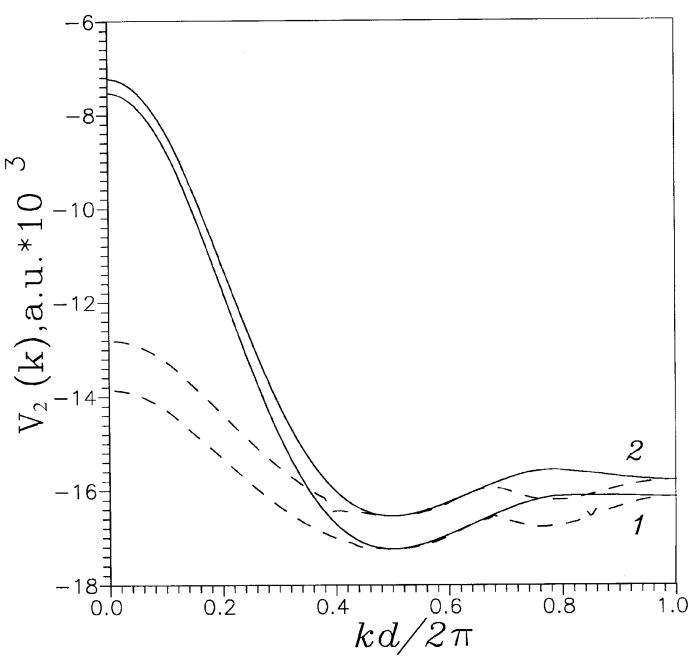

Figure 1. Behaviour of the ordering potential Fourier transform $V_{2}(\boldsymbol{k})$ in the [111] direction in alloys of $K-C s$ system at $T=250 \mathrm{~K}$. Dashed and full curves show results obtained, respectively, with and without the ASD taken into account. Curves 1 refer to alloy $K_{0.7} C s_{0.3}$ while the curves 2 correspond to alloy $K_{0.1} C s_{0.9}$. which is $\tilde{V}_{2}<V_{2}$ in the whole first Brillouin zone except for the points of high symmetry where vector $\boldsymbol{P}_{\boldsymbol{k}}=0$ [5]. One can notice from (32), (33), (20) and (26) that the binary correlation function Fourier components directly depend on the ordering potential, temperature and the ASD. Besides, they are complicated functions of potential $V_{1}$ and the alloy concentration via $\mathrm{cu}-$ mulant $M_{2}$ and the equilibrium atomic volume.

In the present paper the theoretical results are illustrated by numerical calculations performed for the alloys of $K-C s$ and $C a-B a$ systems. Solid solutions of the body centred cubic (bcc) structure exist in wide ranges of temperature and alloy concentration in the both systems [11]. The renormalized potential $\tilde{V}_{2}\left(\boldsymbol{k}, \boldsymbol{A}_{\boldsymbol{k}}\right)(33)$ for $K-C s$ and $C a-B a$ alloys are shown by dashed lines in figures 1 and 2 , respectively. 
The bare ordering potentials $\tilde{V}_{2}(\boldsymbol{k})$ are depicted by full lines. Details of the calculations are omitted because they are the same as those in $[2,5,8]$. The potential $\tilde{V}_{2}\left(\boldsymbol{k}, \boldsymbol{A}_{\boldsymbol{k}}\right)$ has the absolute minimum in the [111] direction in the alloys of the systems investigated. The ASD smooth the dispersion of the ordering potential $V_{2}(\boldsymbol{k})$ in the first Brillouin zone, especially in the [100] direction. It is seen from figures 1 and 2 that an additional minimum appears owing to the ASD in the [111] direction in the alloys of $K-C s$ and $C a-B a$ systems. Potential $\tilde{V}_{2}\left(\boldsymbol{k}, \boldsymbol{A}_{\boldsymbol{k}}\right)(33)$ is one tenth of $\tilde{V}_{2}\left(\boldsymbol{k}, \boldsymbol{A}_{\boldsymbol{k}}\right)$ in $K-C s$ alloys, compare figures 1 and 2. Dependence of $\tilde{V}_{2}\left(\boldsymbol{k}, \boldsymbol{A}_{\boldsymbol{k}}\right)$ on the atomic concentration is more pronounceed in $K-C s$ alloys than in $C a-B a$ ones.

Behaviour of the binary correlation function Fourier components $\left\langle\rho_{\boldsymbol{k}} \rho_{-\boldsymbol{k}}\right\rangle$ in some principal symmetry directions has been investigated according to equation (32) for $K-C s$ and $C a-B a$ alloys. Calculations have been performed with the ASD taken into consideration (dashed curves), and without them: $\boldsymbol{A}_{\boldsymbol{k}} \equiv 0$ for $\boldsymbol{k} \in B Z$ (full curves), see figures 3-6. Drastic effect of the ASD on the $\left\langle\rho_{\boldsymbol{k}} \rho_{-\boldsymbol{k}}\right\rangle=f(\boldsymbol{k})$ behaviour is observed, especially for $C a-B a$ alloys, see figures $3-6$. The ASD encourage the gaining of the $\left\langle\rho_{k} \rho_{-k}\right\rangle$ values in the whole first Brillouin zone. They smooth dispersion of $\left\langle\rho_{\boldsymbol{k}} \rho_{-\boldsymbol{k}}\right\rangle$ in the alloys studied. Thus, the alloys become more similar to the ideal solutions owing to the ASD, especially at high temperatures. The $\left\langle\rho_{\boldsymbol{k}} \rho_{-\boldsymbol{k}}\right\rangle=f(\boldsymbol{k})$ functions strongly depend upon the atomic concentration and temperature in alloys of the both systems investigated, see figures 3-6. Dispersion of $\left\langle\rho_{\boldsymbol{k}} \rho_{-\boldsymbol{k}}\right\rangle$ becomes more

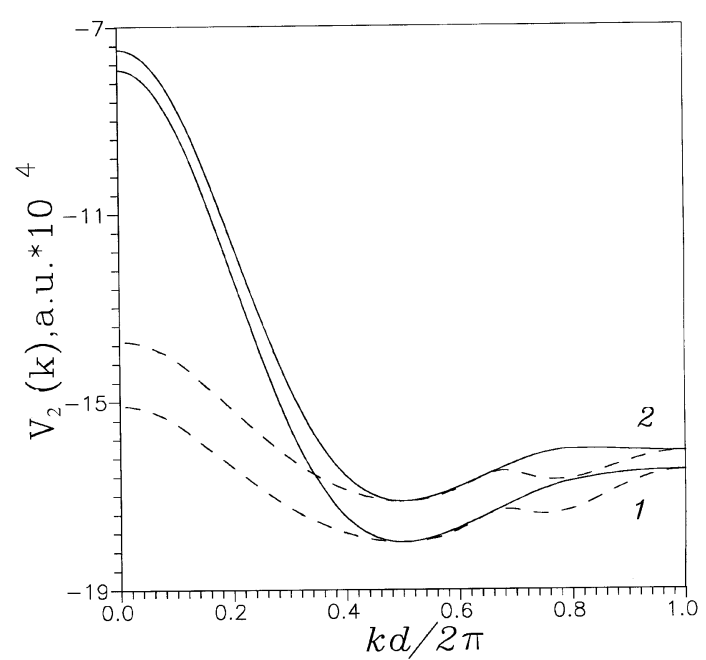

Figure 2. Behaviour of the ordering potential Fourier transform $V_{2}(\boldsymbol{k})$ in the [111] direction in alloys of $C a-B a$ system at $T=750 K$. Notations are the same as in figure 1 . Curves 1 refer to alloy $C a_{0.5} B a_{0.5}$ and the curves 2 correspond to alloy $C a_{0.2} B a_{0.8}$.

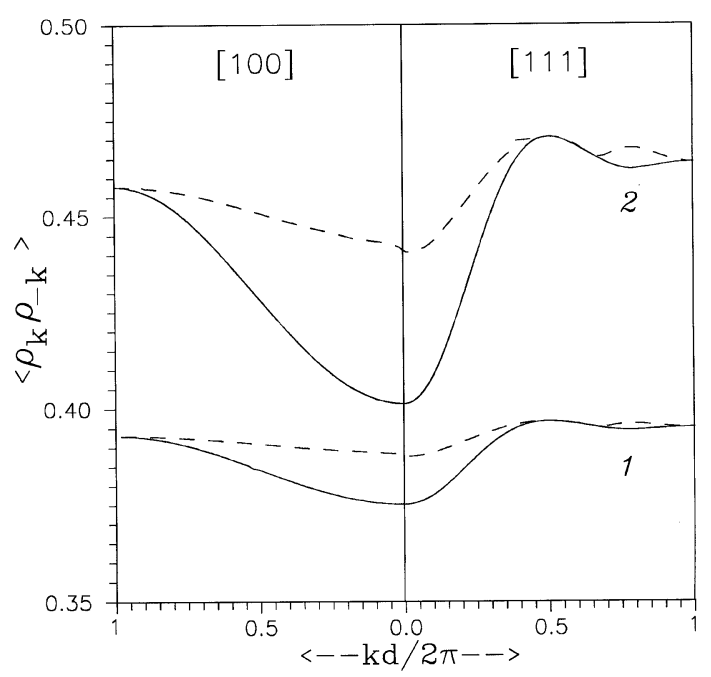

Figure 3. Temperature effects on the binary correlation function Fourier components in the $K_{0.7} C s_{0.3}$ alloy. Dashed and full curves show results obtained, respectively, with and without the ASD taken into account. Curves 1 refer to $T=300 K$ and curves 2 correspond to $T=200 K$. 
visible at the decrease of temperature, see figures $3-5$. The $\left\langle\rho_{\boldsymbol{k}} \rho_{-\boldsymbol{k}}\right\rangle=f(\boldsymbol{k})$ functions display the most interesting behaviour in the [111] direction. The largest effect of the ASD on $\left\langle\rho_{\boldsymbol{k}} \rho_{-\boldsymbol{k}}\right\rangle$ is observed in the [110] direction of the first Brillouin zone, see figures $3-6$.

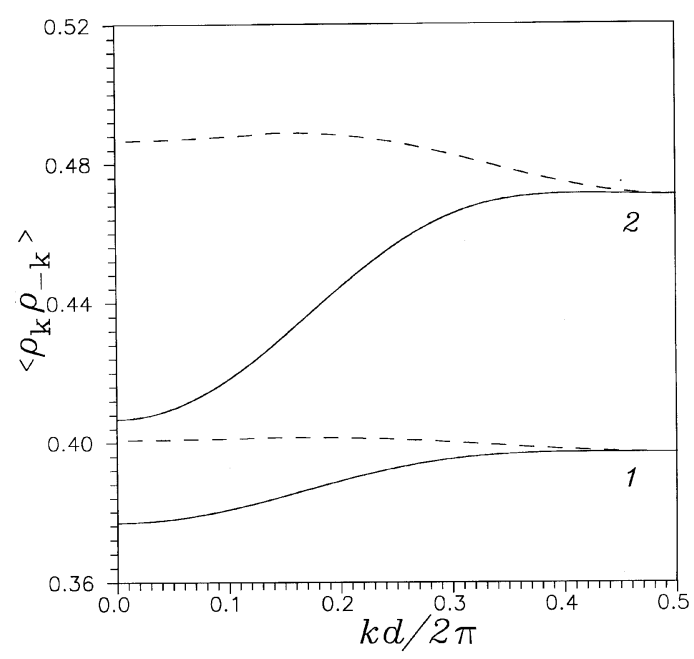

Figure 4. The same for the $K_{0.1} C s_{0.9}$ alloy.
The values of the SRO parameter $\alpha_{R}$ on the first coordination sphere $R_{1}$ have been calculated according to (31). Tables 1 and 2 demonstrate dependence of $\alpha_{R_{1}}$ upon temperature and alloy concentration for $K-C s$ and $C a-B a$ systems. The $\alpha_{R_{1}}$ negative values indicate a trend to ordering in the alloys of the both systems. The ASD favour this tendency: the values of $\alpha_{R_{1}}$ are smaller within the rigid lattice approximation, see tables 1 and 2 . Temperature has a stable effect on $\alpha_{R_{1}}$. The SRO parameter $\alpha_{R_{1}}$ increases with the decrease of temperature. This tendency is most pronounced in alloys with $x=0.65 \div 0.7$ and $x=0.5$ in $K_{x} C s_{1-x}$ and $C a_{x} B a_{1-x}$ systems, respectively, see tables 1 and 2 . The obtained results theoretically agree with the conclusions about the temperature effect on the SRO parameter in alloys, drawn in [3] and based on the experimental investigations of the X-ray diffuse scattering.

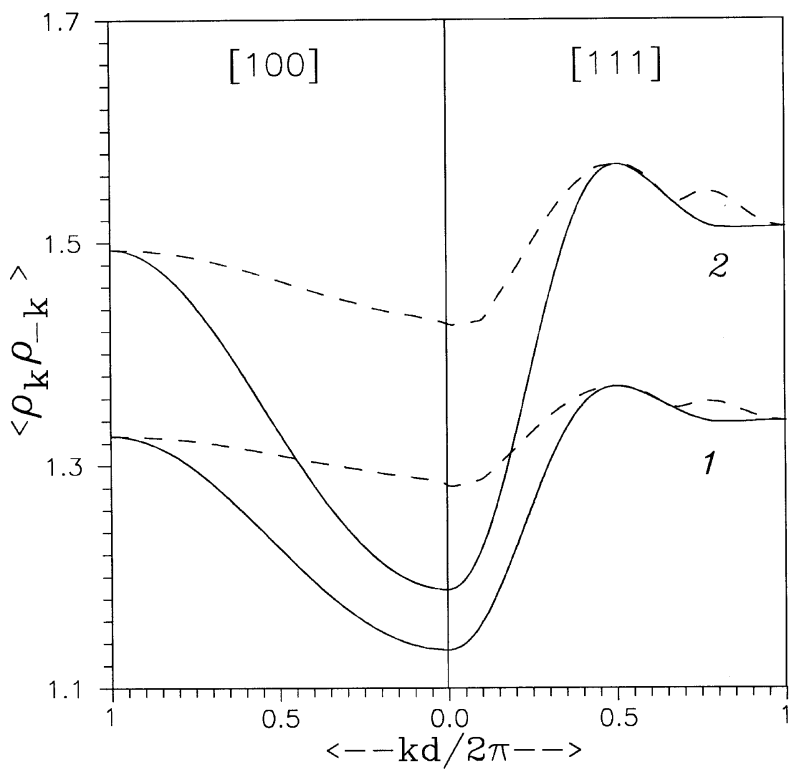

Figure 5. The same for the $C a_{0.5} B a_{0.5}$ alloy. Curves 1 refer to $850 K$ while curves 2 correspond to $T=650 \mathrm{~K}$. 


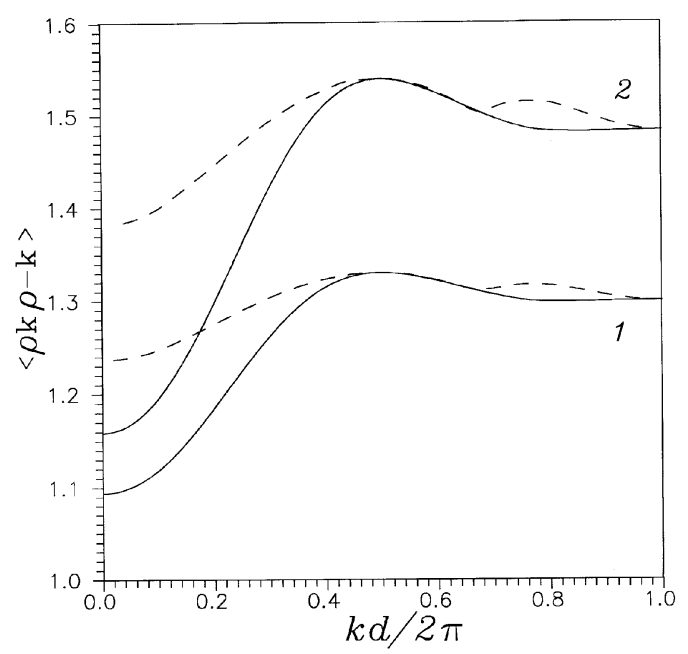

Figure 6. Dependence of the binary correlation function on atomic concentration in the $C a-B a$ system alloys. Direction [111] of the Brillouin zone. Notations are the same as in figure 3. Curves 1 refer to the $C a_{0.2} B a_{0.8}$ alloy and curves 2 correspond to the $C a_{0.8} B a_{0.2}$ alloy.

Table 1. Dependence of the short-range order parameter values on the first coordination sphere upon temperature and alloy concentration in $K_{x} C s_{1-x}$ system. Abreviations RLA and ASD denote respectively that calculations have been performed within the rigid lattice approximation or with the atomic static displacements taken into account.

\begin{tabular}{|c|c|c|c|c|c|c|c|c|c|c|}
\hline \multirow{3}{*}{$\begin{array}{c}\text { Temperature } \\
T, \mathrm{~K}\end{array}$} & \multicolumn{8}{|c|}{ Concentration of potassium, $x$} \\
\cline { 2 - 11 } & \multicolumn{2}{|c|}{0.1} & \multicolumn{2}{|c|}{0.3} & \multicolumn{2}{|c|}{0.5} & \multicolumn{2}{|c|}{0.7} & \multicolumn{2}{c|}{0.9} \\
\cline { 2 - 11 } & RLA & ASD & RLA & ASD & RLA & ASD & RLA & ASD & RLA & ASD \\
\hline 300 & -.216 & -.228 & -.279 & -.294 & -.307 & -.336 & -.298 & -.328 & -.292 & -.281 \\
\hline 250 & -.239 & -.252 & -.298 & -.320 & -.348 & -.379 & -.346 & -.385 & -.337 & -.366 \\
\hline 200 & -.258 & -.272 & -.324 & -.345 & -.378 & -.432 & -.382 & -.453 & -.368 & -.421 \\
\hline
\end{tabular}

Table 2. Values of the short-range order parameter on the first coordination sphere in $C a_{x} B a_{1-x}$ system and their dependence upon temperature and alloy concentration.

\begin{tabular}{|c|c|c|c|c|c|c|c|c|c|c|}
\hline \multirow{3}{*}{$\begin{array}{c}\text { Temperature } \\
T, \mathrm{~K}\end{array}$} & \multicolumn{10}{|c|}{ Concentration of calcium, $x$} \\
\cline { 2 - 11 } & \multicolumn{2}{|c|}{0.1} & \multicolumn{2}{|c|}{0.3} & \multicolumn{2}{|c|}{0.5} & \multicolumn{2}{|c|}{0.7} & \multicolumn{2}{c|}{0.9} \\
\hline 850 & -.203 & -.213 & -.269 & -.281 & -.307 & -.331 & -.298 & -.321 & -.225 & -.226 \\
\hline 750 & -.219 & -.237 & -.287 & -.309 & -.348 & -.376 & -.295 & -.344 & -.238 & -.254 \\
\hline 650 & -.244 & -.264 & -.316 & -.341 & -.378 & -.413 & -.322 & -.352 & -.259 & -.286 \\
\hline
\end{tabular}




\section{Conclusions}

The given results can be summarized in the following statements.

1. The ASD have a drastic effect on the binary correlation function Fourier components $\left\langle\rho_{\boldsymbol{k}} \rho_{-\boldsymbol{k}}\right\rangle$ behaviour in the first Brillouin zone. They smooth the dispersion of the $\left\langle\rho_{\boldsymbol{k}} \rho_{-\boldsymbol{k}}\right\rangle=f(\boldsymbol{k})$-function.

2. Tendency to ordering becomes more pronounced owing to the ASD in the alloys of $K-C s$ and $C a-B a$ systems.

3. Dependence of the SRO parameter on temperature obtained theoretically agrees with the conclusions drawn from the treatment of X-ray diffuse scattering experiments.

\section{Acknowledgements}

The financial support given by the National Academy of Sciences of Ukraine is gratefully acknowledged.

The authors are grateful to Professor I.Stasyuk for interest in this activity and stimulating discussions.

\section{References}

1. Krivoglaz M.A. Theory of X-ray and thermal neutron scattering by real crystals. New York, Plenum, 1989.

2. Yukhnovskii I.R., Gurskii Z.A. Quantum-statistical theory of disordered systems. Kiev, Naukova Dumka, 1991 (in Russian).

3. Iveronova V.I., Katsnelson A.A. Short-range order in solid solutions. Moscow, Nauka, 1977 (in Russian).

4. Hafner J. From Hamiltonians to phase diagrams. Berlin, Springer-Verlag, 1987.

5. Gurskii Z., Khokhlov Yu. Microscopic theory of atomic static displacements in substitutional binary alloys. // J. Phys.: Condens. Matter, 1994, vol. 6, p. 8711-8724.

6. Gurskii Z.A., Chushak Ya. G. Lattice dynamics of binary alloys // Phys. Stat. Sol. (b), 1990, vol. 157, No 2, p. 557-566.

7. Yukhnovskii I.R. Phase transition of the second order. Collective variables method. Singapore, World. Sci. Publ. Co., 1987.

8. Khokhlov Yu., Gurskii Z. A new approach for thermodynamic property investigations of binary alloys. // Metallofizika i Nov. Tekhnol., 1996, vol. 18, No 5, p. 3-12.

9. Hume-Rothery W., Raynor G.V. The structure of metals and alloys. London, Istitute of Metals, 1954.

10. Gurskii Z. Microscopic theory of binary substitional alloys with atomic, static displacements taken into account. // Ukr. Phys. Journ, 1990, vol. 35, No 11, p. 1738-1744 (in Ukrainian).

11. Hansen P.M., Anderko K. Constitution of binary alloys. New York, McGraw-Hill, 1958. 


\section{Статичні зміщення атомів та їхній вплив на близький порядок у сплавах}

\section{3.Гурський, Ю.Хохлов}

Інститут фізики конденсованих систем НАН України, 290011 м. Львів, вул. Свєнціцького, 1

Отримано 26 січня 1998 р.

В рамках мікроскопічної теорії досліджується вплив локальних статичних зміщень атомів (СЗА) на формування близького порядку в бінарних сплавах. Методом колективних змінних отримано явний вираз для Фур'є-компонент бінарної кореляційної функції $\left\langle\rho_{\boldsymbol{k}} \rho_{-\boldsymbol{k}}\right\rangle$. Результати теорії ілюструються числовими розрахунками, виконаними для невпорядкованих сплавів систем K-Cs та Ca-Ва. Спостерігається сильний ефект СЗА на поведінку $\left\langle\rho_{\boldsymbol{k}} \rho_{-\boldsymbol{k}}\right\rangle$-функції у першій зоні Бриллюена. СЗА згладжують дисперсію $\left\langle\rho_{\boldsymbol{k}} \rho_{-k}\right\rangle$-функції. Від'ємні значення параметра близького порядку на першій координаційній сфері вказують на тенденцію до впорядкування у сплавах досліджуваних систем. Показано, що СЗА сприяють цій тенденції. Висновки теорії стосовно впливу температури на параметр близького порядку дуже добре узгоджуються із експериментальними даними, отриманими із обробки дифузного розсіяння рентгенівських променів у бінарних сплавах.

Ключові слова: атомні статичні зміщення, близький порядок, невпорядкований сплав, бінарна кореляційна функція, вільна енергія

PACS: $05.70 . \mathrm{Ce} ; 65.50 .+m$ 